$$
\begin{aligned}
& \text { HePnET } \\
& 1 N O 2
\end{aligned}
$$

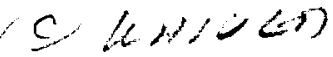

AIAA 2000-0753

\title{
Large Eddy Simulation of Wake Vortices in the Convective Boundary Layer
}

Yuh-Lang Lin, Jongil Han, Jing Zhang, Feng Ding, S. Pal Arya North Carolina State University Raleigh, NC

Fred $\mathrm{H}$. Proctor NASA Langley Research Center Hampton, VA

\section{8th Aerospace Sciences Meeting \& Exhibit January 10-13, 2000 / Reno, NV}

For permission to copy or republish, contact the American Institute of Aeronautics and Astronautics 1801 Alexander Bell Drive, Suite 500, Reston, Virginia 20191-4344 


\title{
LARGE EDDY SIMULATION OF WAKE VORTICES IN THE CONVECTIVE BOUNDARY LAYER
}

\author{
Yuh-Lang Lin ${ }^{*}$, Jongil Han ${ }^{\dagger}$, Jing Zhang ${ }^{\ddagger}$, Feng Ding ${ }^{\S}$, S. Pal Arya \\ Department of Marine, Earth and Atmospheric Sciences \\ North Carolina State University \\ Raleigh, NC 27695-8208 \\ Fred H. Proctorll \\ Airborne Systems Competency \\ NASA Langley Research Center \\ Hampton, VA 23681-2199
}

\begin{abstract}
The behavior of wake vortices in a convective boundary layer is investigated using a validated large eddy simulation model. Our results show that the vortices are largely deformed due to strong turbulent eddy motion while a sinusoidal Crow instability develops. Vortex rising is found to be caused by the updrafts (thermals) during daytime convective conditions and increases with increasing nondimensional turbulence intensity $\eta$. In the downdraft region of the convective boundary layer, vortex sinking is found to be accelerated proportional to increasing $\eta$, with faster speed than that in an ideal line vortex pair in an inviscid fluid. Wake vortices are also shown to be laterally transported over a significant distance due to large turbulent eddy motion. On the other hand, the decay rate of the vortices in the convective boundary layer that increases with increasing $\eta$, is larger in the updraft region than in the downdraft region because of stronger turbulence in the updraft region.
\end{abstract}

\footnotetext{
*Professor, AIAA member

${ }^{\dagger}$ Research Scientist, AIAA member

$\ddagger$ Research Scientist

$\S$ Research Assistant

TProfessor

"Research Scientist, AIAA member
}

This paper is declared a work of the U.S. Government and is not subject to copyright protection in the United States.

\section{Nomenclature}

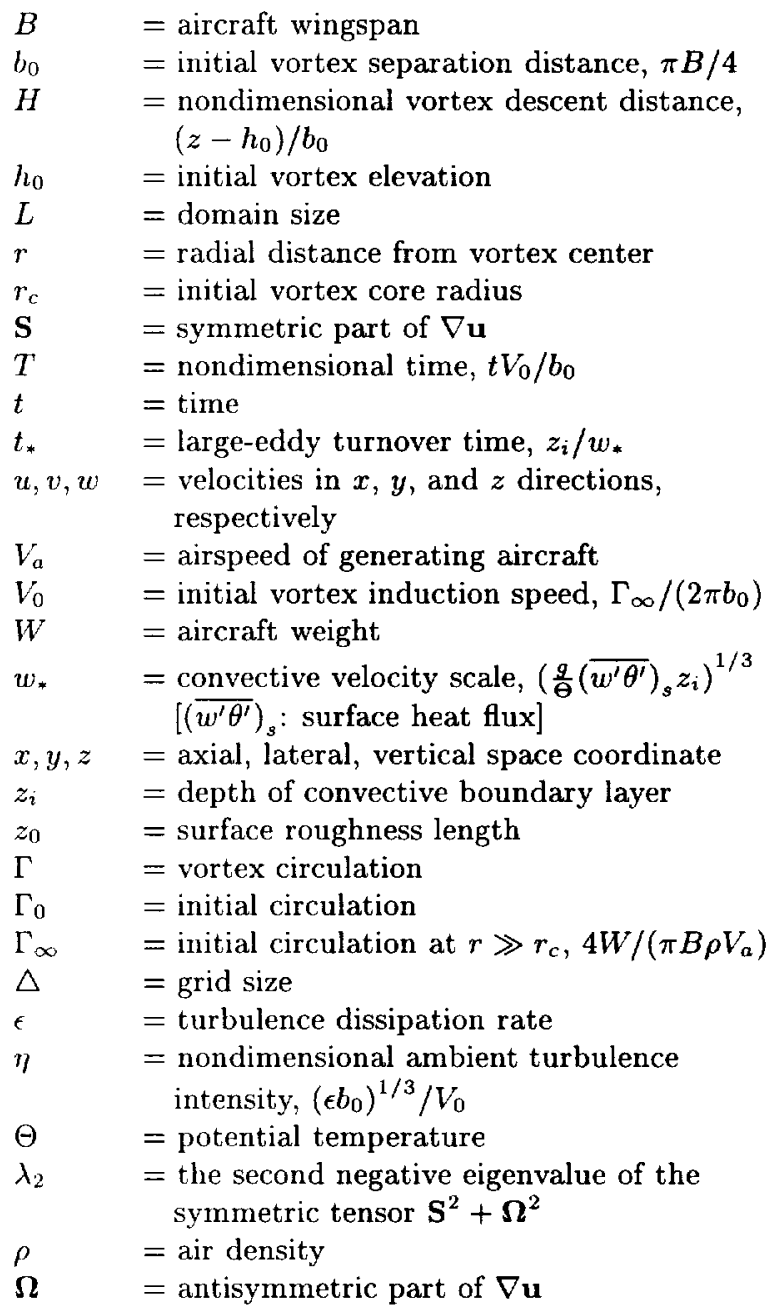




\section{Introduction}

NASA is developing an automated Aircraft Vortex Spacing System ${ }^{1}$ (AVOSS) under its Terminal Area Productivity program. This system will determine safe operating spacing between arriving and departing aircraft as based on ambient weather conditions and provide a safe reduction in separation of aircraft compared to the now-existing flight rules. This system includes prediction algorithms ${ }^{2,3}$ for transport and decay of aircraft wake vortices. To develop this system, research is being focused on understanding the interaction of wake vortices with the atmosphere.

A pair of wake vortices usually descends due to their mutual induction. The vortex descent rate is reduced as the vortices decay with time due to ambient turbulence and stratification. ${ }^{3-5}$ In some cases, however, the vortices can rise. It has been shown ${ }^{6,7}$ that the vortices can either stall or be deflected upwards as they penetrate a strong crosswind shear layer which often occurs just above a developing nocturnal stable layer. Vertical winds associated with weather phenomena such as vigorous fronts, gravity waves, and thermals may also cause the vortices to rise. Rising vortices have been observed during daytime convective conditions, ${ }^{8}$ possibly due to strong thermals developing in the convective boundary layer (CBL). Figure 1 shows some observed vertical position histories of the vortices during typical convective conditions as taken from the Memphis Field Program. ${ }^{9}$ The vortices are observed to remain on the flight level (Fig. 1a), rise (Fig. 1b), or even descend faster than an ideal line vortex pair in an inviscid fluid, for which the sinking rate is given by $d H / d T=-1$ (Fig. 1c). Numerical simulation results by Holzäpfel et al. ${ }^{10}$ indicate that in the CBL some segments of the vortex pair that are placed on an updraft (thermal) area remain on or even rise up to $20 \mathrm{~m}$ above their initial positions. As Holzäpfel et al. pointed out, this situation can be more hazardous for reduced spacing operations due to the increased possibility of encountering stalled vortices during aircraft approach.

The CBL is typically composed of broad regions of gentle downdraft surrounding smaller regions of strong updraft (thermal). The fractional areas are about 0.4 for the updraft and 0.6 for the downdraft. ${ }^{11}$ Both the updraft and downdraft have horizontal scales of roughly $1.5 z_{i}{ }^{12}$ where $z_{i}$ is the CBL depth (of the order of $1 \mathrm{~km}$ ). Vertical velocities in thermals can reach $5 \mathrm{~m} / \mathrm{s}$ or more, although weaker updrafts of 1 to $2 \mathrm{~m} / \mathrm{s}$ are more common. ${ }^{13}$ Therefore, one can expect a large difference in the behavior of wake vortices when they are exposed in the CBL, depending on whether they are placed on the updraft regions or the downdraft regions.

In the present study, we investigate the beliavior of aircraft wake vortices in the CBL using a validated large eddy simulation (LES) model. In Sec. II, we describe the LES model and the initial conditions. In Sec. III,
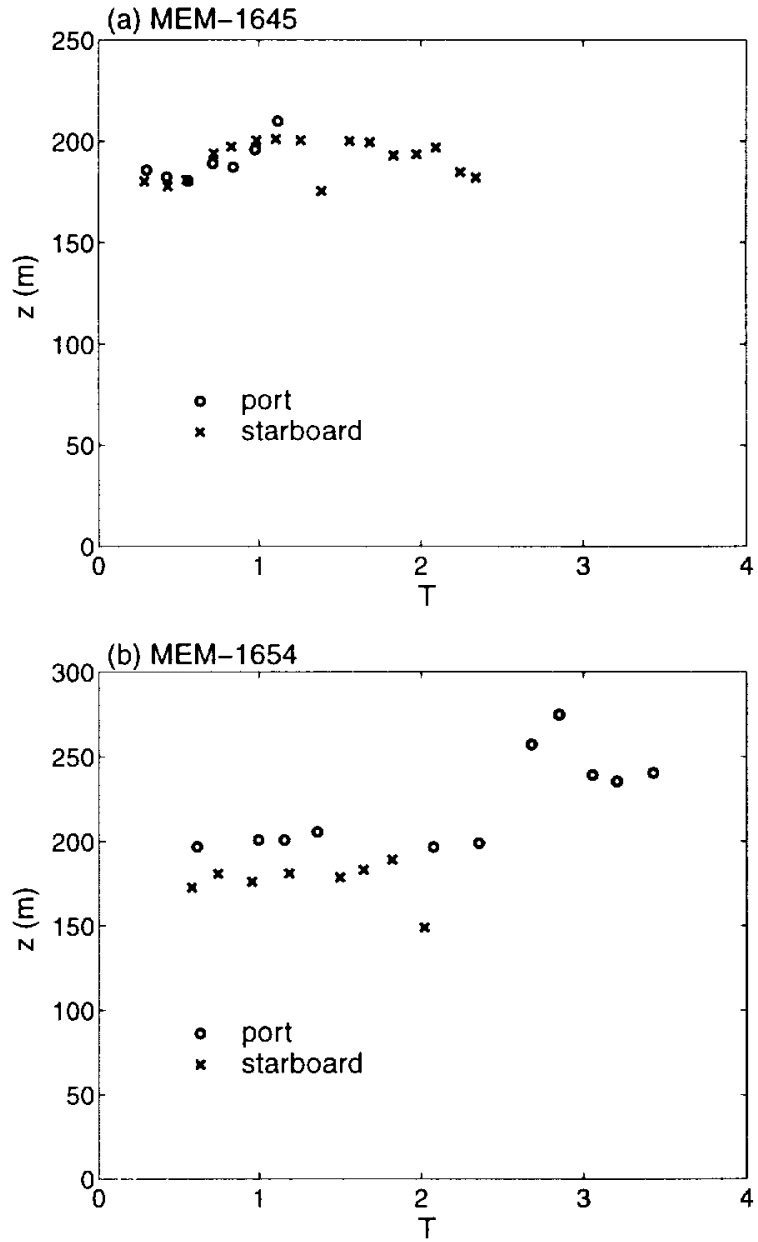

(c) MEM-1583

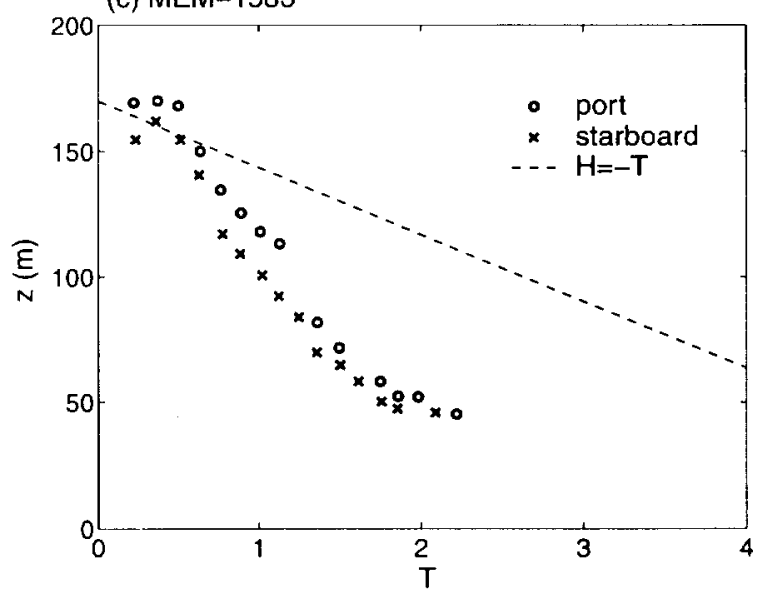

Figure 1: Time evolution of the observed vertical positions of the vortices during daytime convective conditions as taken from the Memphis Field Program. 
we present the results from systematic numerical experiments in terms of nondimensional turbulence intensity. Finally, in Sec. IV, we summarize our results and draw some conclusions.

\section{Model and Initial Conditions}

The numerical model used in the present study is a three-dimensional, nonlinear, compressible, nonhydrostatic LES model, called the Terminal Area Simulation System $^{14}$ (TASS). Numerous successful simulations of interaction of wake vortices with the atmosphere ${ }^{5-7,15-18}$ and some within the $\mathrm{CBL}^{19,20}$ have been conducted with this TASS code. In TASS-LES, grid-scale turbulence is explicitly computed while the effects of subgrid-scale turbulence are parameterized by a conventional first-order closure model with modifications for stratification and flow rotation. ${ }^{5,21}$

The initial vortex system represents a post roll-up, wake-vortex velocity field as described in Proctor, ${ }^{7}$ and consists of a pair of counter-rotating vortices that have no initial variation in the axial direction. This vortex model has also been adopted for investigating the effects of ambient turbulence ${ }^{5}$ and ground ${ }^{16}$ on vortex decay and descent.

One of the major difficulties in numerical simulations of wake vortices is that very fine grid resolution is needed to resolve the vortex core whose size is of the order of $1 \mathrm{~m}$, whereas horizontal domain size of at least four times larger than the CBL height (of the order of $1 \mathrm{~km}$ ) is required to simulate large scale turbulence structure in the CBL. For current computer resources, it is almost impossible to simulate large scale structure of turbulence in the CBL and much smaller scale structure of aircraft wake vortices at the same time. However, the recent study by Holzäpfel et al. ${ }^{10}$ indicates that the main characteristics of vortex behavior in the CBL can be obtained even when greatly reduced domain size (say, about $0.5 \mathrm{~km}$ ) compared to the CBL scale is used.

In this study, in order to achieve a proper resolution of the characteristic scales of both the CBL and the wake vortices, we have chosen a domain size of $\left(L_{x}, L_{y}, L_{z}\right)$ $=(600 \mathrm{~m}, 400 \mathrm{~m}, 350 \mathrm{~m})=\left(15 b_{0}, 10 b_{0}, 8.8 b_{0}\right)$, with a grid size of $(\triangle x, \Delta y, \Delta z)=(5.0 \mathrm{~m}, 2.5 \mathrm{~m}, 2.5 \mathrm{~m})$, where $b_{0}=40 \mathrm{~m}$, and $x, y$ and $z$ correspond to the axial, lateral and vertical directions of the vortex system, respectively. Somewhat larger domain size in the axial direction $\left(15 b_{0}\right)$ can allow the development of Crow instability of which theoretical maximum wavelength is about $8.6 b_{0} \cdot{ }^{22}$ The lateral domain size of $10 b_{0}$ is sufficiently large to minimize boundary influences. The domain size in the vertical direction $\left(8.8 b_{0}\right)$ is large enough to investigate the effects of large turbulent eddies such as thermals on vortex transport. On the other hand, because of our limited computing resources and in order to allow the core to be resolved, the chosen initial core size of $r_{c}=5 \mathrm{~m}$ is somewhat larger than the typical value (less than about $5 \%$ of the wingspan ${ }^{15}$ ) observed behind aircraft. The initial vortices are placed on the height of $h_{0}=180 \mathrm{~m}\left(4.5 \mathrm{~b}_{0}\right)$ above the ground.

Before the vortices are injected, the CBL is driven by a constant, spatially uniform heat flux on the bottom surface until a typical feature of the evolving CBL is well established. Periodic boundary conditions are employed in both horizontal directions. Free-slip and no-slip conditions are imposed at top and bottom boundaries respectively. The Monin-Obukhov similarity relations are used at the surface with surface roughness length, $z_{0}=0.1 \mathrm{~m}$. Initial velocities are specified as zero. Initial potential temperature is vertically uniform. The turbulence is initiated by a small random temperature perturbations superimposed in five lower layers.

The vortices are injected at about 3.6 large-eddy turnover times (defined as $t_{*}=z_{i} / w_{*}$, where $w_{*}$ is the convective velocity scale), when the CBL is well developed although it is still evolving. Figure 2 shows vertical velocity fields on the horizontal $x-y$ plane of the initial vortex elevation and both vertical and lateral velocity fields on the vertical $x-z$ plane along the $y$-center line, with the location of the initial vortex pair indicated. As seen in Fig. 2a and b, the right part of the vortices is placed on an updraft area with stronger vertical velocity, whereas the left part is placed on a downdraft area with weaker vertical velocity. From this vertical velocity distribution, one can expect that the right part of the vortices may stay around the initial vortex elevation or rise, striving against the descent by mutual induction of the vortices, whereas the descent of the left part of the vortices may be accelerated. From Fig. 2c, on the other hand, in lower domain layers below the initial vortex elevation negative lateral velocities are more dominant, and thus, the vortices are expected to be transported mostly to the negative lateral direction during their descent.

To evaluate the sensitivity of vortex behavior to ambient turbulence, we generated five values of nondimensional turbulence strengths of $\eta=0.20,0.25,0.30,0.35$, and 0.50 that are in a typical range of the CBL. These $\eta$ values are typically found during daytime convective conditions and obtained by varying the initial circulation $\left(\Gamma_{\infty}\right)$ to save computing time, rather than by varying initial turbulence field. The associated value of turbulence energy dissipation rate $\epsilon$ is estimated by fitting the theoretical Kolmogorov spectrum in the inertial subrange to the simulated spectrum at the horizontal plane of the initial vortex elevation, as described in Han et al. ${ }^{17}$ Threedimensional wake vortex simulations are carried out with each of the five turbulence field, with all other conditions identical.

\section{Numerical Results}

Figure 3 shows top and side views of the vortex pairs with increasing nondimensional time for three different 

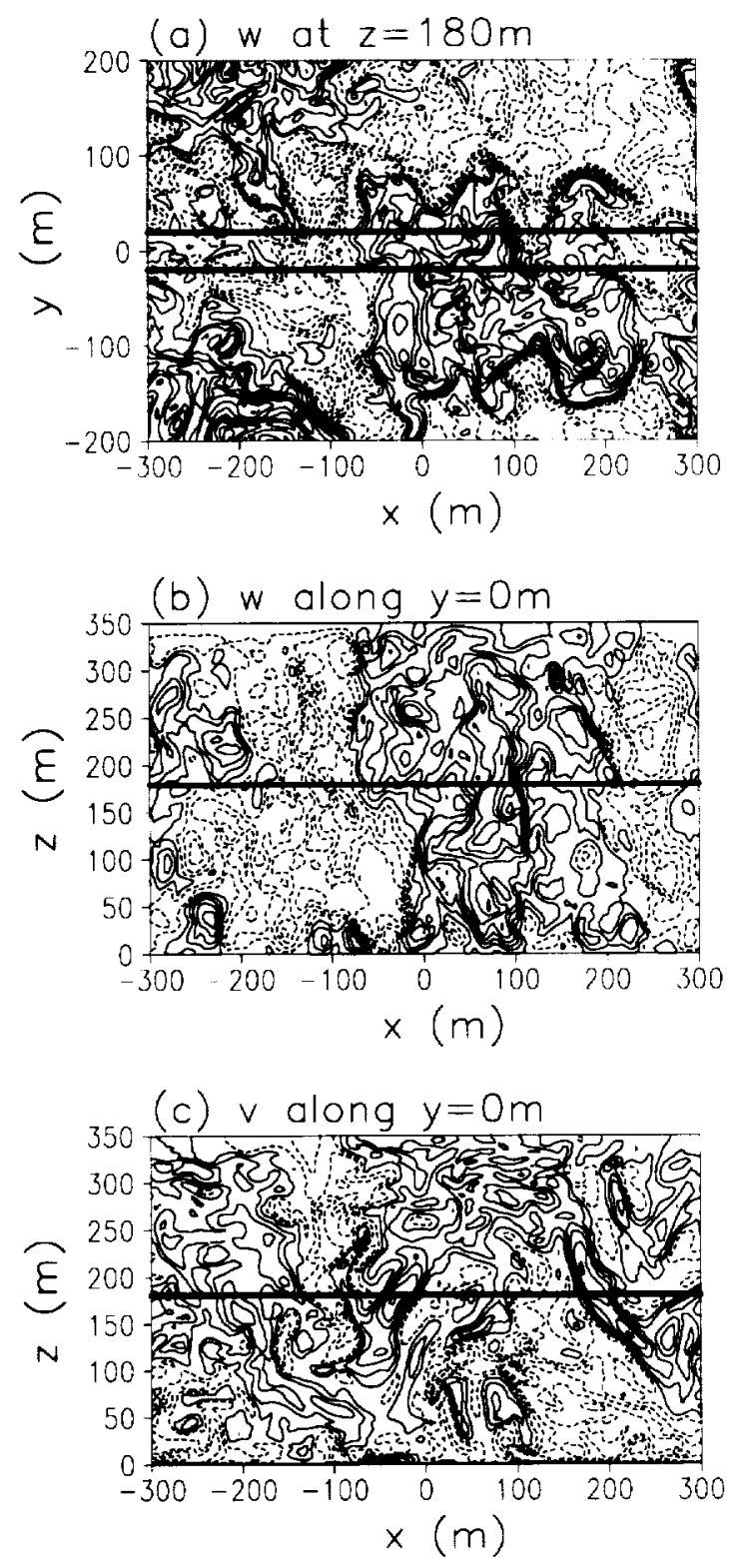

Figure 2: (a) Vertical velocity fields on the horizontal $x-y$ plane of the initial vortex elevation and both (b) vertical and (c) lateral velocity fields on the vertical $x-z$ plane along the $y$-center line, with the location of the initial vortex pair indicated (thick solid line). The contour interval for velocity is $0.4 \mathrm{~ms}$. nondimensional turbulence intensities of $\eta=0.2 .0 .3$. and 0.5 . The method of Jeong and Hussain ${ }^{23}$ is used for the identification of a vortex. They define a vortex in terms of the second negative eigenvalue $\lambda_{2}$ of the symmetric tensor $\mathbf{S}^{2}+\Omega^{2}$, where $\mathbf{S}$ and $\Omega$ are respectively the symmetric and antisymmetric parts of the velocity gradient tensor $\nabla \mathbf{u}$. The top (side) view of Fig. 3 is taken from $x-y(x-z)$ plane projection of the minimum negative $\lambda_{2}$ value along $z(y)$ direction. A threshold $\lambda_{2}$ value to identify the vortex is arbitrarily determined.
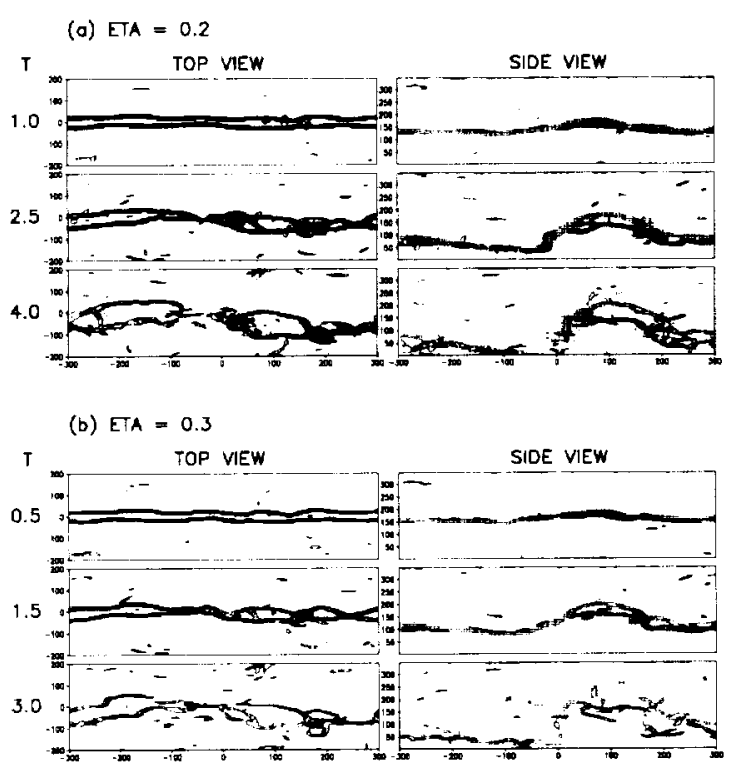

(c) ETA $=0.5$

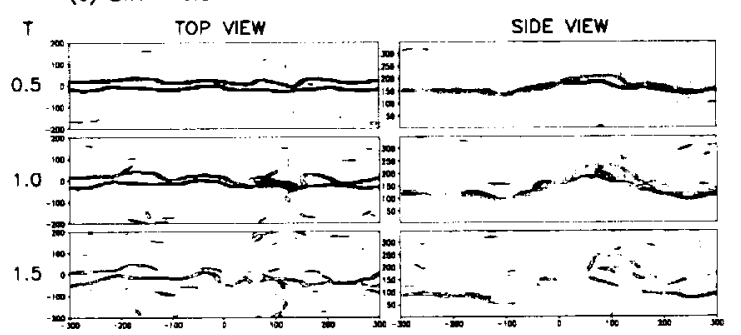

Figure 3: Top and side views for the cases of (a) $\eta=(0.2$, (b) $\eta=0.3$. and (c) $\eta-0.5$. The unit for axis labels is $m$.

As shown in the top view of Fig. 3. because of larger axial domain size $\left(15 b_{0}\right)$ used. a sinusoidal Crow instability develops for all three cases. and then, the vortex pair connects together as a result of the instability and finally disintegrates into a turbulent state. In agreement with Han et al. 's ${ }^{17}$ study, the linking of the vortex pair occurs earlier with stronger turbulence (that is, larger $\eta$ ). In adrlition. the vortices are strongly deformed especially for stronger turbulence due to large turbulent eddy motion. On the other hand. from the side view it is obvious 
that the right part of the vortex pair rises due to strong thermal (updraft) developing in the CBL, whereas the descent of the left part vortex pair accelerates due to the downdraft.

Figure 4 depicts the evolution of maximum vertical vortex positions that are found in the updraft region. It is shown that vortex rise becomes more significant with stronger ambient turbulence strength. For the case of $\eta=0.5$, for example, at $T=1.5$ the vortex rises up to about $2 b_{0}$ from its initial position and its coherent structure is destroyed. For the case of $\eta=0.2$, on the other hand, the vortex appears to stay at its initial height until about $T=2.5$ and then, rises further after its strength becomes much weaker due to dissipation by ambient turbulence.

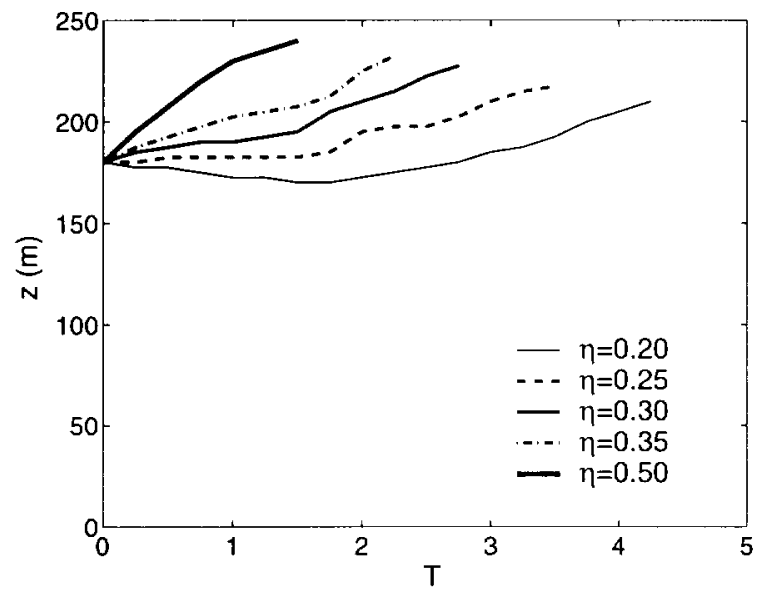

Figure 4: Time evolution of maximum vertical vortex positions for varying nondimensional ambient turbulence strengths.

Figure 5 depicts the evolution of minimum vertical vortex positions that are found in the downdraft region. It is shown that vortex sinking is accelerated due to the downdraft; for all the cases, the sinking rates of the minimum vertical vortex positions are larger than the theoretical sinking rate as given by mutual induction speed of an ideal line vortex pair in an inviscid fluid (that is, $d H / d T=-1$ ). The sinking rates increase with increasing ambient turbulence strength; for $\eta=0.5$ the sinking rate $d H / d T \sim-2.3$.

Figure 6 shows the evolution of vertical vortex positions averaged along the axial direction and their standard deviations. In an average sense, the vortices descend for all $\eta$ values rather than stay or rise as seen in Fig. 6, although they tend to stall at much later times when the vortices almost decay out. This is because the downdrafts not only occupy wider area (about 60\%) than the updrafts, but also the vortex rising due to the updrafts has to counter the effect of sinking due to mutual induction. Unlike the behavior of the minimum vertical

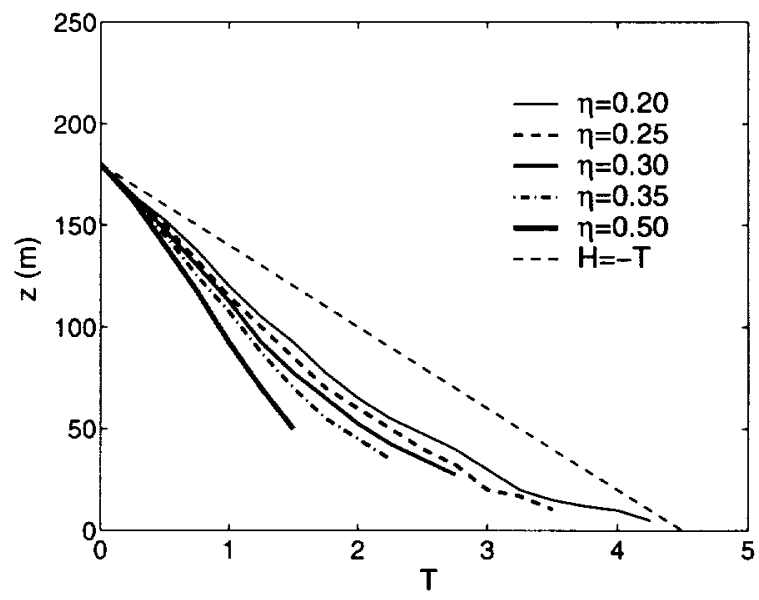

Figure 5: Same as Fig. 4 but for minimum vertical vortex positions.
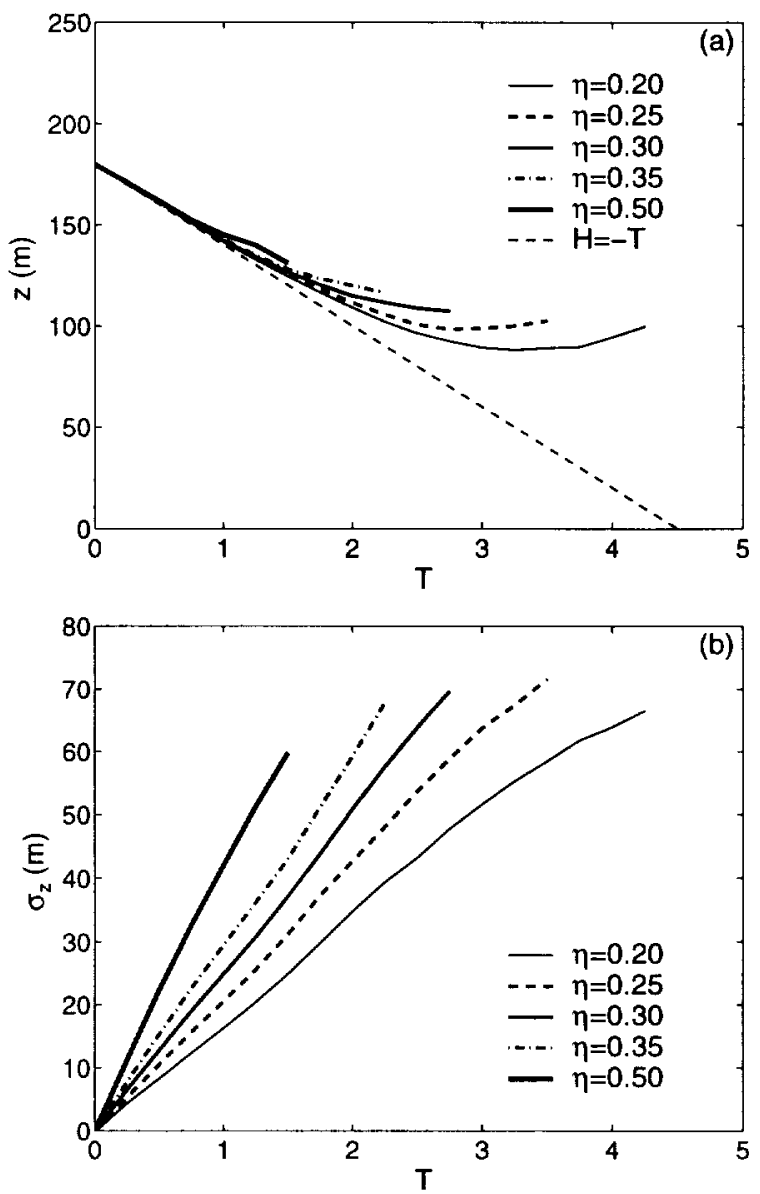

Figure 6: Same as Fig. 4 but for (a) mean vertical vortex positions along the axial direction and (b) standard deviations of vertical vortex positions. 
vortex positions, however, the sinking rates of the average vertical vortex positions are smaller than the theoretical sinking rate of $d H / d T=-1$ and decrease with increasing ambient turbulence strength. On the other hand, the standard deviations of vertical vortex positions increase with increasing ambient turbulence strength, showing about $1.5 b_{0}$ at $T=1.5$ for the extremely strong turbulence case of $\eta=0.5$.

Large turbulent eddies can also transport the vortices laterally over a significant distance, as indicated in the top views of Fig. 3. Figure 7 depicts the evolution of maximum and mean lateral vortex displacements from the initial positions and their standard deviations for five $\eta$ values. Although the maximum and mean lateral displacements and standard deviations appear to increase with increasing ambient turbulence strength, the differences among varying $\eta$ values are much smaller compared with those in vertical positions. Figure 7 a indicates that for the case of $\eta=0.2$, that is, the most long-lived vortex in our experiments, the vortices can be transported up to about $2.5 b_{0}$ at about $T=4$ by large turbulent eddy motion of the CBL.

Figure 8 shows the decay of the average circulation (averaged over a radius between 5 and $15 \mathrm{~m}$ from the center) with time for varying ambient turbulence levels in both updraft (Fig. 8a) and downdraft (Fig. 8b) regions (in each region, the radius-averaged circulations are also averaged over 16 planes $[80 \mathrm{~m}$ ] along the axial direction where the vortex separation is the widest). As shown in Fig. 8, a rapid vortex decay behavior appears to be conspicuous due to buoyancy-driven strong turbulence typical in the CBL. The rate of vortex decay tends to increase with increasing ambient turbulence level, which is consistent with field observations ${ }^{24}$ and our previous numerical simulations. ${ }^{5}$ Vortex decay is more rapid in the updraft region (Fig. 8a) with stronger turbulence strength than in the downdraft (Fig. 8b) region with weaker turbulence strength.

\section{Conclusions}

This study presents the results of numerical simulations which were carried out for understanding how wake vortices behave in the CBL. LES simulations are conducted by introducing a vortex pair, representing aircraft trailing vortices, in a well-developed CBL. Although the domain size chosen is relatively smaller compared to large CBL turbulence scales, the main characteristics of vortex behavior in the CBL seem to be well captured. The following conclusions can be drawn from the results of numerical simulations:

1) In the CBL, the vortices are largely deformed due to strong turbulent eddy motion while a sinusoidal Crow instability develops. Consistent with previous studies, the linking of the vortex pair resulting from the instability occurs earlier with larger nondimensional turbulence intensity.
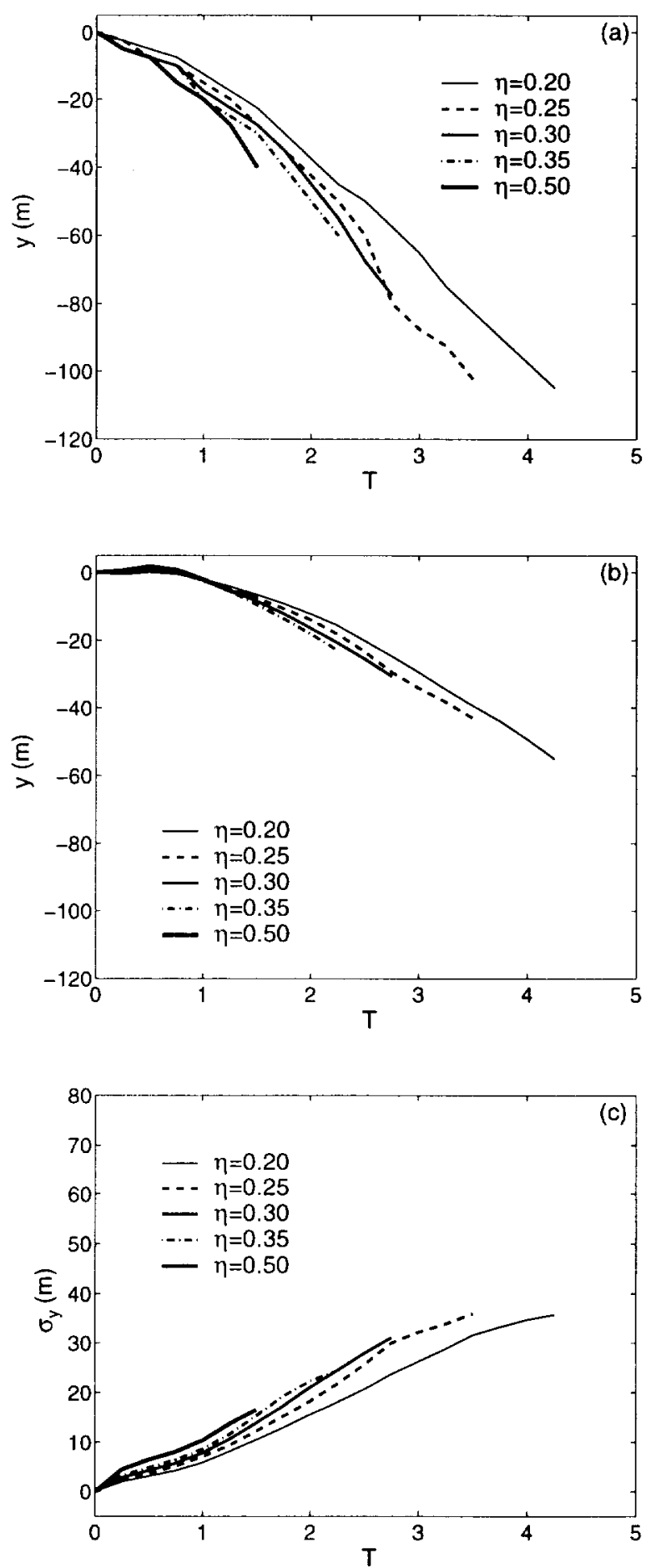

Figure 7: Same as Fig. 4 but for (a) maximum and (b) mean lateral vortex displacements from the initial positions and (c) standard deviations. 
2) Vortex rise is caused by the updrafts (thermals) in the $\mathrm{CBL}$, increases with increasing nondimensional turbulence intensity $\eta$, and can be as large as $H \sim 2$ at $T=1.5$ for the extremely strong turbulence case of $\eta=0.5$.
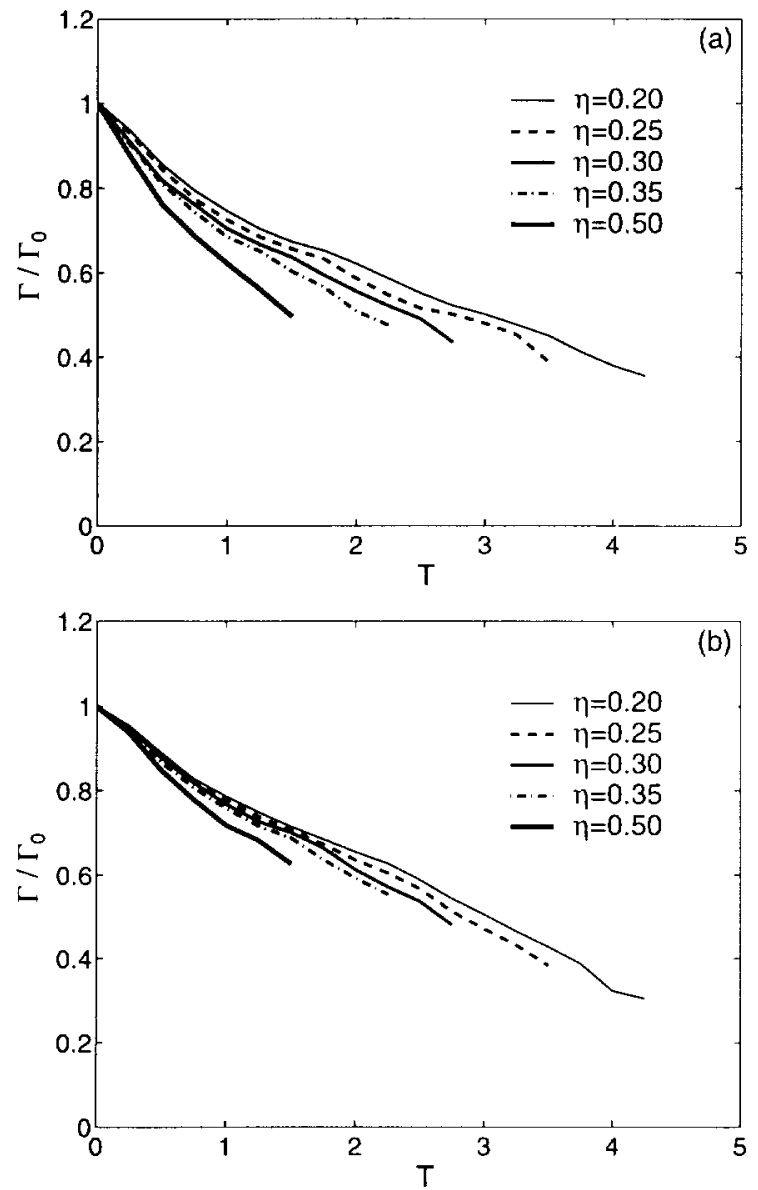

Figure 8: Same as Fig. 4 but for $5-15 m$ averaged circulation in the (a) updraft and (b) downdraft regions.

3) In the downdraft region of the CBL, vortex sinking is accelerated; the sinking rate becomes larger than the theoretical value of $d H / d T=-1$, increases with increasing $\eta$, and can be as large as $d H / d T \sim-2.3$ for $\eta=0.5$.

4) Wake vortices can also be laterally transported over a significant distance due to large turbulent eddy motion in the CBL, and their lateral displacements from the initial positions increase with increasing ambient turbulence strength.

5) Wake vortices in the CBL decay rapidly because of strong turbulence intensity typical in the CBL, whereas the rate of decay increases with increasing $\eta$ and is larger in the updraft region than in the downdraft region.
These results could serve useful for adding a prediction of height and lateral variability in the AVOSS vortex trajectory forecast.

\section{Acknowledgments}

This work was supported by NASA's Terminal Area Productivity program under Contract NAS 1-18925 (Cooperative Agreement NCC-1-188). Numerical simulations were carried out on NASA's Cray C90 and J90 and North Carolina Supercomputing Center's Cray T916.

\section{References}

${ }^{1}$ Hinton, D. A., Charnock, J. K., Bagwell, D. R., and Grigsby, D., "NASA Aircraft Vortex Spacing System Development Status," NASA TM-110184, August 1995. 37th Aerospace Sciences Meeting \& Exhibit, Reno, NV, AIAA Paper No. 99-0753, January 1999.

${ }^{2}$ Robins, R. E. and Delisi, D. P., "Further Development of a Wake Vortex Predictor Algorithm and Comparisons to Data," 37th Aerospace Sciences Meeting \& Exhibit, Reno, NV, AIAA Paper No. 99-0757, January 1999 .

${ }^{3}$ Sarpkaya, T., "A New Model for Vortex Decay in the Atmosphere," 37th Aerospace Sciences Meeting \& Exhibit, Reno, NV, AIAA Paper No. 99-0761, January 1999.

${ }^{4}$ Greene, G. C., "An Approximate Model of Vortex Decay in the Atmosphere," Journal of Aircraft, Vol. 23, No. 7, 1986, pp. 566-573.

${ }^{5}$ Han, J., Lin, Y. -L., Arya, S. P., and Proctor, F. H., "Numerical Study of Wake Vortex Decay and Descent in Homogeneous Atmospheric Turbulence," In press, AIAA Journal, Vol. 38, 2000.

${ }^{6}$ Proctor, F. H., Hinton, D. A., Han, J., Schowalter, D. G., and Lin, Y. -L., "Two-Dimensional Wake Vortex Simulations in the Atmosphere: Preliminary Sensitivity Studies," 35th Aerospace Sciences Meeting \& Exhibit, Reno, NV, AIAA Paper No. 97-0056, January 1997.

${ }^{7}$ Proctor, F. H., "The NASA-Langley Wake Vortex Modeling Effort in Support of an Operational Aircraft Spacing System," 36th Aerospace Sciences Meeting \& Exhibit, Reno, NV, AIAA Paper No. 98-0589, January 1998.

${ }^{8}$ Zak, J. A. and Rodgers, Jr., W. G., "Documentation of Atmospheric Conditions During Observed Rising Aircraft Wakes," NASA Contractor Report 4767, 1997.

${ }^{9}$ Campbell, S. D., Dasey, T. J., Freehart, R. E., Heinrichs, R. M., Matthews, M. P., Perras, G. H., and Rowe, G. S., "Wake Vortex Field Measurement Program at Memphis, TN Data Guide," Project Report: NASA/L-2, January 1997. [Available from NTIS]

${ }^{10}$ Holzäpfel, F., Gerz, M., Frech, M., and Dornbrack, A., "The Decay of Wake Vortices in the Convective Boundary Layer," 37th Aerospace Sciences Meeting \& 
${ }^{11}$ Weil, J. C., "Dispersion in the Convective Boundary Layer," Lectures on Air Pollution Modeling, A. Venkatram and J. C. Wyngaard, Eds., Amer. Metcor. Soc., Boston, 1988, pp. 167-227.

${ }^{12}$ Caughey, S. J., Kitchen M., and Leighton, J. R., "Turbulence Structure in Convective Boundary Layers and Implications for Diffusion," Boundary-Layer Meteorol., Vol. 25, 1983, pp. 345-352.

${ }^{13}$ Stull, R. B., An Introduction to Boundary Layer Meteorology, Kluwer Academic Publishers, Dordrecht, $1988,666 \mathrm{pp}$.

${ }^{14}$ Proctor, F. H., "The Terminal Area Simulations System, Volume 1: Theoretical Formulation," NASA Contractor Report 4046, DOT/FAA/PM-86/50, 1, 1987.

${ }^{15}$ Proctor, F. H., "Numerical Simulation of Wake Vortices Measured During the Idaho Fall and Memphis Field Programs," 14th AIA A Applied Aerodynamics Conference, Proceedings, Part-2, New Orleans, LA, AIAA Paper No. 96-2496, June 1996. pp. 943-960.

${ }^{16}$ Proctor, F. H. and Han, J., "Numerical Study of Wake Vortex Interaction with the Ground Using the Terminal Area Simulation System," 37th Aerospace Sciences Meeting \& Exhibit, Reno, NV, AIAA Paper No. 99-0754, January 1999.

${ }^{17}$ Han, J., Lin, Y. -L., Schowalter, D. G., Arya, S. P., and Proctor, F. H., "Large Eddy Simulation of Aircraft Wake Vortices within Homogeneous Turbulence: Crow Instability," In press, AIAA Journal, Vol. 38, 2000.

${ }^{18}$ Schowalter, D. G., Decroix, D. S., Switzer, G. F,, Lin, Y. -L., and Arya, S. P., "Toward Three-Dimensional Modeling of a Wake Vortex Pair in the Turbulent Boundary Layer," 35th Aerospace Sciences Meeting \& Exhibit, Reno, NV, AIAA Paper No. 97-0058, January 1997.

${ }^{19}$ Schowalter, D. G., DeCroix, D. S., Proctor, F. H., Lin, Y.-L., Arya, S. P., and Kaplan, M. L., "Turbulent Statistics in the Atmospheric Boundary Layer: A Comparison of Large Eddy Simulation with Observations," 11th Symposium on Boundary Layers and Turbulence, Charlotte, NC, March 1995, pp. 552-555.

${ }^{20}$ Schowalter, D. G., DeCroix, D. S., Lin, Y.-L., Arya, S. P., and Kaplan, M. L., "Planetary Boundary Layer Simulation Using TASS," NASA Contractor Report 198325, 1996, $41 \mathrm{pp}$.

${ }^{21}$ Proctor, F. H., "A LES Subgrid Turbulence Model with Rotational Dampening," Submitted as NASA Technical Report.

${ }^{22}$ Crow, S. C., "Stability Theory for a Pair of Trailing Vortices," AIAA Journal, Vol. 8, No. 12, 1970, pp. 2172-2179.

${ }^{23}$ Jeong, J. and Hussain, F., "On the Identification of a Vortex," Journal of Fluid Mechanics, Vol. 285, 1995, pp. 69-94.

${ }^{24}$ Sarpkaya, T., "Decay of Wake Vortices of Large Aircraft," AIAA Journal, Vol. 36, No. 9, 1998, pp. 